Meta

Journal des traducteurs

Translators' Journal

\title{
La traduction comme création littéraire
}

\section{Jaume Pérez Muntaner}

Volume 38, numéro 4, décembre 1993

Le Je du traducteur

The $I$ of the Translator

URI : https://id.erudit.org/iderudit/003681ar

DOI : https://doi.org/10.7202/003681ar

Aller au sommaire du numéro

Éditeur(s)

Les Presses de l'Université de Montréal

ISSN

0026-0452 (imprimé)

Découvrir la revue

Citer cet article

Pérez Muntaner, J. (1993). La traduction comme création littéraire. Meta, 38(4), 637-642. https://doi.org/10.7202/003681ar d'utilisation que vous pouvez consulter en ligne.

https://apropos.erudit.org/fr/usagers/politique-dutilisation/ 


\section{LA TRADUCTION COMME CRÉATION LITTÉRAIRE}

JAUME PÉREZ MUNTANER

Université de Valence, Valence, Espagne

Il n'est pas facile d'appliquer, dans le domaine de la théorie ou de la réflexion, à une expérience de traducteur, une activité littéraire qui, dans la plupart des cas, se définit surtout comme pratique. C'est la pratique qui fait et définit le traducteur, et c'est elle aussi qui l'aide à résoudre les innombrables problèmes qui se présentent à lui, même si ces derniers sont presque tous d'origine philologique, linguistique ou littéraire, ou en rapport avec des connaissances aussi variées que les œuvres traduites qui, partant, sont aussi liées à la géographie, à l'histoire, à la religion ou à l'expérience directe de la vie quotidienne.

Ce qui précède ne signifie pas cependant que théorie et praxis apparaissent dissociées quand on traduit, mais nous travaillons ou nous traduisons fréquemment à partir de présupposés théoriques inconscients, non formalisés, s'appuyant sur de vagues schémas méthodologiques qui sont variables, s'adaptant ou se configurant peu à peu dans le processus même de la traduction. En tout cas, toute traduction qui se veut sérieuse et responsable suppose, en plus d'une réflexion constante et de décisions indispensables pour réaliser le passage d'une langue à une autre, un certain nombre de considérations théoriques, initiales ou finales.

On peut ainsi considérer que nous avons, d'une part, le fait de choisir un texte écrit en une langue différente et de prendre la décision de le traduire - entreprise qui suppose une stratégie de travail déterminée, consciente ou non de la part du traducteur. D'autre part, nous avons la pratique quotidienne de la traduction, avec les problèmes innombrables qui se présentent au jour le jour, page après page, et qu'il faut résoudre au fur et à mesure, depuis le choix des acceptions considérées les plus justes jusqu'à la recherche d'une formulation plus en accord avec les caractéristiques de la langue d'arrivée, ou au respect et à la conservation d'une syntaxe qui, bien qu'étrangère à la langue d'arrivée, peut s'avérer, à un moment donné, plus ferme, plus efficace et/ou plus fidèle. Finalement, une fois que le passage de la langue de départ à celle du traducteur a été réalisé, une réflexion sur les résultats obtenus s'impose. C'est le moment de l'évaluation, de la lecture finale du nouveau texte, qui lime les inexactitudes, élimine les erreurs, polit les phrases peu heureuses; moment qui est certainement celui où le traducteur peut faire preuve de la plus grande créativité, car il représente l'ultime tentative pour arriver à doter le texte de possibilités et de caractéristiques semblables à celles du texte de départ.

Je pars ici d'une expérience limitée comme traducteur, fondamentalement de l'anglais en catalan, avec quelques incursions du côté italien comme langue de départ et du côté espagnol comme langue d'arrivée. Mon activité est centrée presque exclusivement sur ce qu'on appelle la traduction litteraire : traductions de poésie et de roman. Ces deux genres sont ceux qui peuvent conditionner une différence première et décisive dans mes considérations sur la traduction: je veux parler de la différence qui existe, en règle générale, entre le vers et la prose, entre un langage lyrique, subjectif, hautement connoté et soumis à des contraintes formelles, et un autre, à intention plus objective, plus descriptive, moins lié aux possibilités rythmiques et associatives qui caractérisent le vers. 
Mon intérêt pour la traduction a toujours été étroitement uni à celui que j'ai aussi pour la littérature. En conséquence, je considère, dans une certaine mesure, la traduction comme une prolongation de mon travail d'écrivain. Dans mes traductions de poésie, il est clair que ce qui a été déterminant, c'est d'abord pour moi, l'identification avec quelques aspects ou quelques caractéristiques de l'auteur, ou, plus souvent, avec ses poèmes. Ensuite, il y a le désir de les faire connaître dans ma propre langue, car je suis conscient de l'enrichissement littéraire potentiel que cette activité suppose, tant d'un point de vue personnel que collectif. Je pense, en effet, que la traduction peut être - ou est toujours réellement - une fenêtre ouverte sur d'autres cultures, un souffle rénovateur qui aide à moderniser et à revitaliser notre propre tradition littéraire. Sans cette rénovation, sans cette revitalisation, la culture et la littérature d'un pays, et tout spécialement si cette littérature occupe un espace restreint comme la Catalogne, courraient le risque de se scléroser. Heureusement, depuis la fin du XIX ${ }^{\mathrm{e}}$ siècle, nous avons eu, dans le domaine de la traduction, une volonté ferme manifeste déjà dans ce texte de Joan Maragall, qui date de 1893 :

Quand le travail de traduction se fait avec une véritable chaleur artistique, il suggère des formes nouvelles. Il fait découvrir, dans la langue, des richesses qui y étaient inconnues et il lui apporte vigueur et souplesse. La qualité de la traduction lui confère aussi de la dignité et pallie en grande mesure, l'absence de continuité dans notre propre tradition littéraire. En outre, la traduction met notre langue en contact avec l'esprit humain universel et la fait marcher dans la même direction ${ }^{1}$.

Traduire se transforme ainsi en une activité vitale pour la modernisation de notre propre littérature, grâce à un très large échange d'idées, de formes et de propositions, une ouverture sans réserves, qui est «contraste de tous les élans vivants, unité humaine désintéressée que nous recherchons au moyen de méthodes convergentes et complémentaires ${ }^{2} \gg$, comme le disait un autre grand poète catalan, Josep Carner, en parlant du rôle de la tradition dans la culture.

Dans cette perspective, je n'hésite pas à avouer que je considère mes traductions de poésie comme une continuation de mon œuvre de poète, ni à dire que, parfois, elles ont eu - et ont - une influence sur ma propre poésie, sur ma conception de l'œuvre littéraire et, en général, sur les textes que j'écris. Bref, pour m'exprimer d'une manière encore plus nette, ce sont mes créations et je peux les placer sur le même plan que le reste de mes poèmes. Elles m'appartiennent en catalan presque autant qu'elles appartiennent - pour citer deux exemples proches parce que je les ai traduits - à E.E. Cummings ou à Anne Sexton en leur version originale anglaise. De fait, la décision de les traduire et de me les approprier, de les faire miens et, dans une certaine mesure, de les transformer et de les trahir, est redevable à moi seul. En outre, la difficulté a été probablement aussi grande pour moi, au moment de la recréation, que pour les auteurs au moment de la création. Je crois même que, sous certains aspects, elle a peut-être été plus grande pour moi, car le traducteur de poésie, qui s'oblige volontairement à la recréation dans une autre langue, essaie d'être tout à fait fidèle ou - au moins - de ne pas trop trahir. Il est, de cette manière, constamment limité par les effets, par la forme, par le contenu et même par les éventuels défauts ou les formulations peu heureuses du poème original.

J'ai été frappé, lorsque j'ai réalisé mes premiers travaux en collaboration avec le poète américain William Stafford, sur les textes de poètes catalans, par le fait que le résultat final paraissait dans les revues The Paris Review et Grilled Flowers comme s'il s'agissait d'œuvres de William Stafford, et que l'on n'indiquait, qu'en caractères minuscules ou entre parenthèses, le nom de l'auteur original : Vicent Andrés Estellès, ou celui de Vance Savage qui avait collaboré à la traduction, ou le mien propre. En fait, c'était Stafford qui leur donnait la forme ultime et définitive, qui les faisait siens et les poétisait dans sa propre 
langue, en respectant le plus possible les vers originaux. Il pouvait ainsi s'attribuer de plein droit la création ou recréation des poèmes dans leur version anglaise, sans trahir l'original et sans se trahir lui-même comme poète.

Peu de temps après, j'ai découvert comment Robert Lowell s'appropriait d'une manière toute naturelle, dans Notebook, des vers bien connus de Bécquer: «Volverán las oscuras golodrinas» (Les noires hirondelles reviendront...) et les transformait en un poème à la saveur indubitablement lowellienne :

The dark swallows will doubtless come back here killing the injudicious nightflies with a clack of the beak; but these that stopped in flight to see your beauty and my good fortune... as if they knew our names - they'll not come back. The thick lemony honeysuckle, climbing from its earthroot to jour window, will open more beautiful blossoms to the evening; but these... dewdrops, trembling, shining, falling, the tears of the day - they'll not come back. Yet love will sound its fireword to you, and wake your heart, perhaps, from its deep sleep; but silent, absorbed, and on their knees, as men adore God at the altar, as I love you - don't be deceived, you will not be loved like that ${ }^{3}$.

J'ai voulu reproduire entièrement le poème de Lowell pour montrer combien est étroite la relation de dépendance qu'il entretient avec les vers de Bécquer et combien sa traduction en est, dans l'ensemble, littérale. Il s'agit bien évidemment d'un emprunt parfaitement licite en poésie et, plus généralement, en littérature. Ainsi le poème de l'écrivain romantique espagnol, comme ce sonnet bien connu, de Quevedo: «Miré los muros de la patria mía» (J'ai regardé vers les murs de ma patrie), également traduit par Lowell, est devenu, dans la version anglaise, un poème de l'écrivain américain. En fin de compte, traduire un texte est la meilleure manière, la plus directe, de le lire, de l'interpréter et, en conséquence, de le trahir de manière intelligente, c'est-à-dire de se l'approprier et de le convertir en sa propre poésie. La lecture, comme l'interprétation et la trahison, sont les conditions de la création littéraire.

D'une manière attendue, après ce que je viens de dire, il est parfaitement logique qu'un poète comme Joan Ferraté, lequel est aussi critique littéraire, ne veuille pas renoncer à ses traductions de poésie et les considère partie intégrante de sa propre œuvre; ou encore, que dans les œuvres complètes de Marià Vilangómez, des versions poétiques de différents auteurs étrangers soient introduites - procédé que j'approuve totalement. Il s'agit en effet, et sans qu'il soit possible d'en douter, de véritables travaux de création, et il est probable qu'une communion totale a existé, au(x) moment(s) de leur écriture, avec un texte traduit et que celui-ci peut seulement être comparé au moment de la création poétique initiale et à celui de son inspiration. Il ne s'agit donc pas d'une appropriation illicite ou indue, mais du résultat d'un immense effort qui ne veut pas passer inaperçu et rester simplement entre les limites du plus pur altruisme. George Steiner a défini le phénomène avec précision:

Undoubtedly translation contains a paradox of altruism - a word on which there are stresses both of "otherness" and of "alteration". The translator performs for others, at the price of dispersal and relative devaluation, a task no longer necessary or immediate to himself. But there is also a proprietary impulse. It is only when he "brings home" the simulacrum of the original, when he recrosses the divide of language and community, that he feels himself in authentic possession of his source ${ }^{4}$.

Le sentiment de possession est si fort que le traducteur se sent souvent possesseur de l'ouvre traduite, auteur d'un nouveau texte en une langue différente, surtout si la décision de la traduction lui revient entièrement et qu'elle n'a été conditionnée par aucune exigence d'édition. Il naît ainsi une relation intime et étonnante entre le traducteur et l'auteur original, ou, mieux encore, entre le nouveau poète et le poème qu'il a lu, qu'il a pensé 
traduire et qu'il a finalement traduit. Cette relation apparaît au moment même de la lecture, de la lecture sérieuse profonde et on peut en conclure une vérité incontestable: lire est, au fond, traduire et la traduction, comme je l'ai laissé entendre auparavant, est la manière la plus parfaite, la plus complète de lire. Josep Carner l'écrivait, en 1944, en des termes plus chargés d'émotion, de lyrisme et de vérité que les miens: «Traduire une œuvre est la meilleure manière de la lire. C'est aimer et souffrir, c'est la servir et la dominer. ${ }^{5}$ » En fin de compte, comme l'a dit Harold Bloom, «une personne ne lit que ce qu'elle est», ajoutant peu après: «une personne est ou devient ce qu'elle lit». Ces mots confirment donc la relation intime entre le lecteur et sa lecture et, aussi, la faculté de création que le lecteur accorde au texte choisi, de telle sorte qu'on peut dire que l'acte de lecture profonde est le lieu de la réalisation d'une authentique création - ou recréation - du texte poétique. Dans la traduction, celle-ci est menée à ses dernières conséquences : le traducteur assimile le texte lu et le transforme en quelque chose qui lui appartient déjà complètement.

En vérité, c'est cette sensation que je ressens personnellement en présence d'une grande partic de mes traductions poétiques. Elles sont tout simplement mes créatures et elles n'existeraient pas sans ma volonté arrêtée de les lire, de les écrire ou de les traduire et, finalement, de les publier. Je peux dire en toute tranquillité que ce sont mes poèmes, bien que ces poèmes proviennent directement de l'œuvre d'autres poètes, ou en nuançant, pour être moins radical, que je me sens coauteur et cocréateur de ces poèmes.

Je soulignerai cependant, que mon attitude vis-à-vis de la traduction poétique est, sous quelques aspects, différente de celle adoptée quand il s'agit de traduction en prose. Dans les deux cas, l'idée directrice est celle de la plus grande fidélité possible au texte original. Je sais que la fidélité totale n'existe pratiquement pas, mais l'œuvre en prose - le genre narratif moins conditionnée par les aspects formels, parce qu'elle est plus analytique, plus objective que l'œuvre lyrique, peut plus facilement être fidèle. Pour ces mêmes raisons et toujours d'une manière générale, l'ceuvre en prose présente normalement moins de difficulté de compréhension, ce qui facilite l'exégèse et les phases suivantes de la recherche de l'équivalence qui sont, dans toute tentative de traduction, la compréhension, la reformulation et la justification, comme le fait remarquer Jean Delisle 6 .

Et, de fait, dans mon expérience de traducteur, j'ai toujours eu moins de difficultés avec les œuvres en prose et, généralement, ces difficultés ont été limitées à la seule obligation de trouver, dans la langue d'arrivée, l'équivalence plus ou moins exacte pour les expressions figées, les phrases faites, les formulations syntaxiques ou les conversations en slang, ou, encore, à l'obligation d'adapter tout ceci en catalan, quand j'estimais que l'adaptation serait plus expressive et plus enrichissante que les équivalences que pouvait me fournir ma langue. Telle a été ma pratique habituelle dans la traduction de The Bean Tree (1991) de Barbara Kingsolver et - dans une moindre mesure, parce que quelqu'un m'avait précédé pour quelques narrations, avec l'excellente version de Carles Riba - dans la traduction de quelques contes de Edgar Alan Poe, intitulée, selon un critère imposé par la maison d'édition, Ciència ficció (1988).

Malgré ce que je viens de dire, la traduction n'est jamais facile et suppose une dose considérable de stupeur et de souffrance; stupeur et souffrance devant l'impossibilité de surmonter l'incompréhension partielle d'une phrase ou d'une situation, ou devant l'immense difficulté qu'il y a à trouver les équivalences les mieux adaptées, rarement exactes dans la langue du traducteur. Il s'agit de difficultés qui, en principe, se retrouvent toutes, dans une même mesure, dans la traduction littéraire. Mais s'il y a un comble de la souffrance, il doit être dans la traduction de la poésie, dans la lutte, presque aussi diabolique que la création littéraire elle-même, pour reproduire, dans une langue différente, une œuvre artistique reposant sur les spécificités de la langue de l'auteur. C'est pour cette raison 
que je considère si justes et si vrais les mots que j'ai déjà cités de Carner : «Traduire, surtout traduire de la poésie, c'est avant tout l'aimer, la souffrir, l'asservir et la dominer.»

Je peux m'imaginer moi-même - ou imaginer une autre personne - traduisant un roman qui ne suscite pas l'enthousiasme, qui intéresse peu les lecteurs, mais il me serait difficile de réussir une très bonne traduction d'un poème avec lequel je n'ai pas d'affinités profondes. Cette traduction pourrait probablement être, en apparence, fidèle et didactique, mais le lyrisme, la spécificité poétique du texte original, se réduirait considérablement dans le passage d'une langue à l'autre. Manquerait la première des caractéristiques signalées par Carner: aimer le texte, le comprendre, avec cette compréhension dont Delisle disait qu'elle était la condition indispensable de la pratique de la traduction, avec la souffrance, intimement liée à l'amour, à la difficulté de compréhension qui peut naître à chaque vers.

Traduire signifie aussi servitude, idée en relation aussi avec celle d'amour et de fidélité. Le traducteur doit savoir se maintenir dans le juste milieu et dire seulement ce qui se trouve dans le texte original. La tentation de l'infidélité est grande et les difficultés sont immenses, parce qu'il est souvent impossible de reproduire, dans la langue d'arrivée, la multiplicité des registres, les références et les connotations d'un poème. Dans la plupart des cas, il faut savoir choisir et mettre en évidence dans le poème les éléments les plus caractéristiques et les plus personnels du texte original, mais je pense que, généralement, l'idéal de la fidélité la plus absolue doit être la règle d'or de toute bonne traduction. Deux exemples de E.E. Cummings, apparemment simples, peuvent illustrer ma volonté de «coller» au texte et, en même temps, mon désir d'obtenir le meilleur rendement possible dans la langue d'arrivée. Je donne ces exemples pour montrer que, si l'on tente d'être fidèle, il est possible d'aboutir à des résultats assez semblables à ceux de l'original.

\begin{tabular}{llll}
1 & 1 & 2 & 2 \\
\hline l(a & s(una & un (bee) mo & im (abella) pa \\
le & fu & vi & ssi \\
af & lla & n (in) g & bl (en) e \\
fa & c & are (the) you (only) & ú) tu (nica) \\
ll & a & & adorm (rosa) ida \\
s) & u) & asl (rose) eep & \\
one & oli & & \\
l & t & & \\
iness & ud & &
\end{tabular}

Un mot finalement sur la question de la maîtrise du texte. Ce terme, pour Carner, renvoie probablement à l'idée de la compréhension totale du texte à traduire, mais cette compréhension, cette connaissance profonde, implique la maîtrise d'une matière qui paraît ardue et semble difficile à obtenir. La maîtrise signifie possession ou désir de possession, de la même manière qu'on désire posséder tout ce qui a constitué un enjeu, un défi, ce qui nous a servi et nous sert de stimulation et ce dans quoi on a dépensé une grande partie de notre énergie, au moins autant - sinon plus - que celle dont le poète a eu besoin pour écrire son «propre» poème. La traduction littéraire, et surtout la traduction de la poésie, est, et peut être, une œuvre de création, une œuvre pleine de servitudes qui ne peut ni ne doit remplacer l'œuvre originale, mais qui, dans certains cas, peut atteindre un pouvoir de suggestion semblable à celui qui existait dans la langue de départ. Le mérite - ou presque 
tout le mérite - doit en revenir alors au traducteur qui est, en définitive et sans qu'on puisse en douter, l'auteur du résultat final.

\section{(Traduction de Brigitte Lépinette)}

\section{Notes}

1. Joan Maragall, Obres Completes, vol I, Barcelone, Editorial Selecta, 1970, p. 789.

2. Joseph Carner, Teoría de l' ham poètic, Barcelone, Edicions 62, 1970, p. 93.

3. Robert Lowell, Notebook, New York, Farrar, Straus and Giroux, 1971, p. 210.

4. George Steiner, After Babel. Aspects of Languages and Translation, Oxford, Oxford University Press, 1975 , p. 380.

5. Cité par Albert Manent Josep Carner i el Noucentisme Barcelone, Edicions 62, 1969, p. 186

6. Jean Delisle, L'analyse du discours comme méthode de traduction: initiation a la traduction française de textes pragmatiques anglais, Ottawa, Presses de 1'Université d'Ottawa, 1980, p. 65. 